\title{
A CHARACTERIZATION OF THE LEAST CARDINAL FOR WHICH THE BAIRE CATEGORY THEOREM FAILS
}

\author{
ARNOLD W. MILLER
}

\begin{abstract}
Let $\kappa$ be the least cardinal such that the real line can be covered by $\kappa$ many nowhere dense sets. We show that $\kappa$ can be characterized as the least cardinal such that "infinitely equal" reals fail to exist for families of cardinality $\kappa$.
\end{abstract}

Let Baire $(\kappa)$ stand for:

"The real line is not the union of $\kappa$ many nowhere dense sets

(a set is nowhere dense iff its closure has no interior)".

The property was extensively studied in Miller (1981) and Miller (1982). It is easily seen (see Kuratowski (1966)) that: not Baire $(\kappa)$ iff some separable, completely metrizable space is the union of $\kappa$ many nowhere dense sets iff every separable, completely metrizable space without isolated points is the union of $\kappa$ many nowhere dense sets.

For example, we may replace the real line by Cantor space $\left(2^{\omega}\right)$, or Baire space $\left(\omega^{\omega}\right)$. Recall that $\omega$ is the first infinite ordinal and is equal to its set of predecessors (i.e. the nonnegative integers), $2=\{0,1\}, X^{Y}$ is the set of functions mapping $Y$ into $X, 2$ and $\omega$ have the discrete topology, and $2^{\omega}$ and $\omega^{\omega}$ have the product topology.

Let Uniformity $(\kappa)$ stand for the proposition

"Every subset of the real line of cardinality less than or equal to $\kappa$ is meager (a set is meager iff it is the union of countably many nowhere dense sets)".

Let us recall some standard terminology: $|X|$ is the cardinality of $X$, (for any cardinal $\kappa)[X]^{\kappa}=\{Y|Y \subseteq X| Y \mid,=\kappa\},[X]^{\leqslant \kappa}=\{Y|Y \subseteq X| Y \mid, \leqslant \kappa\}, \forall^{\infty} n$ abbreviates "for all but finitely many $n$ ", and $\exists^{\infty} n$ abbreviates "there are infinitely many $n$ ".

Consider the following properties:

$$
\begin{aligned}
& \text { Different ( }(\kappa) \text { iff } \forall A \in\left[\omega^{\omega}\right]^{\leqslant \kappa} \exists X \in[\omega]^{\omega} \exists f \in \omega^{\omega} \forall g \in A \\
& \forall^{\infty} n \in X f(n) \neq g(n) \text {; } \\
& \text { Equal }(\kappa) \text { iff } \forall A \in\left[\omega^{\omega}\right]^{\leqslant \kappa} \forall B \in\left[[\omega]^{\omega}\right]^{\leqslant \kappa} \exists f \in \omega^{\omega} \forall g \in A \\
& \forall X \in B \exists^{\infty} n \in X(f(n)=g(n)) \text {. }
\end{aligned}
$$

Received by the editors April 20, 1981.

AMS (MOS) subject classifications (1970). Primary 02K05.

${ }^{1}$ Research partially supported by an NSF Grant. 
In Miller (1981) it was shown that Uniformity $(\kappa)$ iff Different $(\kappa)$. A less satisfactory property was found equivalent to Baire $(\kappa)$. The purpose of this note is to prove

THEOREM. Baire $(\kappa)$ iff Equal $(\kappa)$.

To see that Baire $(\kappa)$ implies Equal $(\kappa)$, note that for any $g \in \omega^{\omega}$ and $X \in[\omega]^{\omega}$, $\left\{f \in \omega^{\omega} \mid \forall n \in X f(n) \neq g(n)\right\}$ is closed nowhere dense in $\omega^{\omega}$.

Now let us prove that Equal $(\kappa)$ implies Baire $(\kappa)$. Let Independent $(\kappa)$ stand for:

$$
\text { “ } \forall B \in\left[[\omega]^{\omega}\right]^{\leqslant \kappa} \exists Z \in[\omega]^{\omega} \forall X \in B|X \cap Z|=|X-Z|=\omega " .
$$

LEMMA 1. Equal $(\kappa)$ implies Independent $(\kappa)$.

Proof. Let $B=\left\{X_{\alpha} \mid \alpha<\kappa\right\}$ and choose $X_{\alpha}^{i} \in\left[X_{\alpha}\right]^{\omega}$ for $i=0,1$ so that $X_{\alpha}^{0} \cap X_{\alpha}^{1}$ $=\varnothing$. Choose $g_{\alpha}^{i} \in \omega^{\omega}$ so that $g_{\alpha}^{i}\left(X_{\alpha}^{i}\right)=\{i\}$. By Equal $(\kappa)$ let $f \in \omega^{\omega}$ be such that for all $\alpha$ and $i, \exists^{\infty} n \in X_{\alpha}^{i} f(n)=g_{\alpha}^{i}(n)=i$. Then $Z=f^{-1}\{0\}$ does the job.

Definition. $Z \in[\omega]^{\omega}$ is $l$-uncrowded iff $\forall n, m \in Z n \neq m \rightarrow|n-m| \geqslant l$.

Definition. l-Uncrowded $(\kappa)$ iff $\forall B \in\left[[\omega]^{\omega}\right]^{\leqslant \kappa} \quad \exists Z$ l-uncrowded $\forall X \in B$ $|X \cap Z|=\omega$.

LEMMA 2. The following are equivalent.

(A) Independent $(\kappa)$,

(B) 2-Uncrowded $(\kappa)$,

(C) for all $l<\omega$ l-Uncrowded $(\kappa)$.

Proof. Let us first prove that (A) implies (B). I claim there exists $T \in[\omega]^{\omega}$ such that for every $X \in B,|X-T|=\omega$. To see this, note that Independent $(\kappa)$ implies $\kappa<c=\left|\omega^{\omega}\right|$. A well-known theorem of Sierpinski (1928) says that there exists an almost disjoint family of cardinality $c$, i.e. there exists $M_{\alpha} \in[\omega]^{\omega}$ for $\alpha<c$ such that for all $\alpha \neq \beta,\left|M_{\alpha} \cap M_{\beta}\right|<\omega$. Since the $M_{\alpha}$ are almost disjoint and $\kappa<c$, for some $\alpha<c$ for all $X \in B\left|X-M_{\alpha}\right|=\omega$. Let $T$ be any such $M_{\alpha}$.

Let $E$ be the even integers and $O$ the odd integers. Without loss of generality we may assume that for all $X \in B, X \subseteq E-T$ or $X \subseteq O-T$. Let $T=\left\{a_{n}: n<\omega\right\}$ be an enumeration in increasing order and for any $a$ and $b$ let $(a, b)=\{n \in \omega \mid a<n$ $<b\}$. For each $X \in B$ let $X^{*}=\left\{n \mid\left(a_{n}, a_{n+1}\right) \cap X \neq \varnothing\right\}$. Let $W$ be independent with respect to $\left\{X^{*} \mid X \in B\right\}$ (i.e. for all $X^{*},\left|X^{*}-W\right|=\left|X^{*} \cap W\right|=\omega$ ). Let

$$
Z=\cup\left\{\left(a_{n}, a_{n+1}\right) \cap E \mid n \in W\right\} \cup\left\{\left(a_{n}, a_{n+1}\right) \cap O \mid n \notin W\right\} .
$$

It is easily checked that $Z$ is 2 -uncrowded and for all $X \in B,|Z \cap X|=\omega$.

(B) implies (C) is proved by induction on $l$. Suppose $Z$ is $l$-crowded and for all $X \in B,|X \cap Z|=\omega$. Let $Z=\left\{a_{n}: n<\omega\right\}$ (increasing order) and for each $X \in B$, $X^{*}=\left\{n \mid a_{n} \in X\right\}$. Let $Q$ be a 2-uncrowded set such that for all $X \in B$, $\left|X^{*} \cap Q\right|=\omega$. Then $\left\{a_{n} \mid n \in Q\right\}$ is a $2 l$-uncrowded set meeting each element of $B$ in an infinite set.

Now we prove (B) implies (A). Let $\left\{W_{\alpha} \mid \alpha<\kappa\right\} \subseteq[\omega]^{\omega}$. For each $\alpha<\kappa$, let $W_{\alpha}^{e}=\left\{2 n \mid n \in W_{\alpha}\right\}$ and $W_{\alpha}^{0}=\left\{2 n+1 \mid n \in W_{\alpha}\right\}$. Let $Z$ be a 2-uncrowded set such that for each $\alpha<\kappa,\left|Z \cap W_{\alpha}^{e}\right|=\left|Z \cap W_{\dot{\alpha}}^{0}\right|=\omega$. Let $Q=\{n \mid 2 n \in Z\}$. Then 
$\left|Z \cap W_{\alpha}^{e}\right|=\omega$ implies $\left|Q \cap W_{\alpha}\right|=\omega$, and $\left|Z \cap W_{\alpha}^{0}\right|=\omega$ implies $\left|W_{\alpha}-Q\right|=\omega$ since $2 n+1 \in Z$ implies $2 n \notin Z$.

LEMMA 3. (Equal ( $\kappa)) \forall F \in\left[\omega^{\omega}\right]^{\leqslant \kappa}$ there exists a sequence $n_{k}<\omega$ for $k<\omega$ such that $n_{k+1}>\sum_{i=0}^{k} n_{i}$ and for every $f \in F, \exists^{\infty} k f\left(n_{k}\right)<n_{k+2}$.

Proof. We may assume without loss of generality that each $f \in F$ is strictly increasing. Choose $g \in \omega^{\omega}$ such that for every $f \in F, \exists^{\infty} n g(n)=f(n)$. Construct a sequence $n_{k}$ for $k<\omega$ so that $n_{k+1}>\sum_{i=0}^{k} n_{i}$ and for every $i \leqslant n_{k}, g(i)<n_{k+1}$. Then for every $f \in F \exists^{\infty} k f\left(n_{k}\right)<n_{k+2}$.

Now we finish proving the theorem. Let us review some standard terminology. Let $2^{<\omega}=\cup_{n<\omega} 2^{n}$ and for $s \in 2^{<\omega}$ let $|s|$ be the length of $s$ (i.e. that $n$ such that $s \in 2^{n}$ ). For $s$ and $t$ elements of $2^{<\omega}$ let $s^{\wedge} t$ be their concatenation. A basic clopen subset of $2^{\omega}$ is of the form $[s]=\left\{x \in 2^{\omega} \mid s \subseteq x\right\}$ for some $s \in 2^{<\omega}$.

Suppose $D_{\alpha} \subseteq Z^{\omega}$ for $\alpha<\kappa$ are dense open sets. We must show that $\cap_{\alpha<\kappa} D_{\alpha} \neq \varnothing$. Construct $f_{\alpha}: \omega \rightarrow 2^{<\omega}$ such that for every $s \in 2^{<n},\left[s^{\wedge} f_{\alpha}(n)\right] \subseteq D_{\alpha}$. This is done by successively extending $\left|2^{\leqslant n}\right|$ times. By Lemma 3 there exists a sequence $n_{k}$ for $k<\omega$ with $n_{k+1}>\sum_{i=0}^{k} n_{i}$ and for each $\alpha, \exists^{\infty} k\left|f_{\alpha}\left(n_{k}\right)\right|<n_{k+2}$. By Equal $(\kappa)$ there exists $g: \omega \rightarrow 2^{<\omega}$ such that for each $\alpha<\kappa$,

$$
X_{\alpha}=\left\{k<\omega \mid f_{\alpha}\left(n_{k}\right)=g(k) \text { and }\left|f_{\alpha}\left(n_{k}\right)\right|<n_{k+2}\right\}
$$

is infinite. We may assume that for all $k,|g(k)|<n_{k+2}$. Now let $Z$ be a 3-uncrowded set such that for all $\alpha<\kappa,\left|X_{\alpha} \cap Z\right|=\omega$ and let $Z=\left\{k_{n} \mid n<\omega\right\}$. Define $h \in 2^{\omega}$ to be the infinite concatenation

$$
g\left(k_{0}\right) \hat{g}\left(k_{1}\right) \hat{g} g\left(k_{2}\right) \hat{\ldots} .
$$

Then $h \in D_{\alpha}$ for each $\alpha$, because if $k_{n} \in Z \cap X_{\alpha}$, then

$$
\left|g\left(k_{0}\right) \hat{g}\left(k_{1}\right) \hat{\cdots} \hat{g}\left(k_{n-1}\right)\right| \leqslant \sum_{i=0}^{n-1} n_{k_{1}+2} \leqslant n_{k_{n-1}+3} \leqslant n_{k_{n}}
$$

and $g\left(k_{n}\right)=f_{\alpha}\left(n_{k_{n}}\right)$.

The notion of independent family is due to Fichtenholz and Kantorovitch (1934). The property Independent $(\kappa)$ is due to R. Price (1979). The notion of uncrowded set is new here, therefore let us scrutinize some variations of it.

First, we may weaken this notion by saying that $Z \subseteq \omega$ is loosely packed iff there exists $N<\omega$ such that for all $i,(i, i+N)-Z \neq \varnothing$ (i.e. $Z$ does not contain a block of $N-1$ consecutive integers). Call a set $Z \in[\omega]^{\omega} \infty$-uncrowded iff $Z=\left\{a_{n}\right.$ : $n<\omega\}$ and $\lim _{n \rightarrow \infty}\left(a_{n+1}-a_{n}\right)=\infty$. Define the two properties Loosely packed $(\kappa)$ and $\infty$-Uncrowded $(\kappa)$ by requiring that for every $B \in\left[[\omega]^{\omega}\right]^{<\kappa}$ there exists $Z$ loosely packed ( $\infty$-uncrowded) such that for all $X \in B,|Z \cap X|=\omega$.

THEOREM. (A) Independent ( $\kappa$ ) iff Loosely packed $(\kappa)$.

(B) Independent $\left(\omega_{1}\right) \neq \infty$-Uncrowded $\left(\omega_{1}\right) \neq$ Baire $\left(\omega_{1}\right)$.

To prove part (A) left to right, just note that a 2-uncrowded set is loosely packed. Now suppose that Independent $(\kappa)$ fails. Then there exists $B \in\left[[\omega]^{\omega}\right]^{<\kappa}$ such that for every finite partition of $\omega,\left\{X_{0}, X_{1}, X_{2}, \ldots, X_{n-1}\right\}$, there exists $i<n$ and $X \in B$ 
such that $X \subseteq X_{1}$. The easiest way to obtain such a B is by a Lowenheim-Skolem argument. (Those readers unfamiliar with the logic involved are invited to find their own proof.) Let $B_{0} \in\left[[\omega]^{\omega}\right]^{<\kappa}$ witness that Independent $(\kappa)$ fails, i.e. $\forall Z \in[\omega]^{\omega}$ $\exists X \in B_{0} X \cap Z$ is finite or $X-Z$ is finite. Let $M$ be an elementary substructure of $\left(H\left(c^{+}\right), \varepsilon\right)$ such that $\left\{B_{0}\right\} \cup B_{0} \subseteq M$ and $|M| \leqslant k$. Let $B=M \cap[\omega]^{\omega}$. For any $Z \in M \cap[\omega]^{\omega}$ and $Y \subseteq Z$ there exist $X \in M \cap[\omega]^{\omega}$ such that $X \subseteq Z$ or $X \cap Y=$ $\varnothing$. This is because there is, in $M$, a bijection between $Z$ and $\omega$. Now by an easy induction on $n<\omega$, for every partition $X_{0}, X_{1}, X_{2}, \ldots, X_{n-1}$ of $\omega$ suppose $Z$ is loosely packed and $N$ is such that for every $i,(i, i+N)-Z \neq \varnothing$. Define $X_{l}$ for $l<N$ by $k \in X_{l}$ iff $(k \cdot N+l) \notin Z$. Clearly $\cup_{l<N} X_{l}=\omega$. So $\exists Q \in B, \exists l<N Q \subseteq$ $X_{l}$. But then $\{k \cdot N+l \mid k \in Q\} \cap Z=\varnothing$, so not Loosely packed $(\kappa)$.

Next we show that Independent $\left(\omega_{1}\right)$ does not imply $\infty$-Uncrowded $\left(\omega_{1}\right)$. This fact is demonstrated by the random real model of Solovay. This model is obtained by forcing with the measure algebra $B$ of $2^{\kappa}$ for some $\kappa \geqslant \omega_{2}$ over a model of $\mathrm{CH}$. One easily shows that if $R$ is a random subset of $\omega$ then for every $X \in[\omega]^{\omega}$ in the ground model, $|X-R|=|X \cap R|=\omega$. Now suppose

$$
\mu(\llbracket \tau \subseteq \omega \wedge \tau \infty \text {-uncrowded } \rrbracket)=1 .
$$

By standard arguments obtain an increasing sequence $n_{k}$ in the ground model such that $\mu\left(\llbracket \forall k\left(\tau-n_{k}\right)\right.$ is $k$-uncrowded $\left.\rrbracket=c\right) \geqslant \frac{1}{2}$. Letting $b_{i}=\llbracket i \in \tau \rrbracket$ we see that for any $i<j<k, \mu\left(b_{n_{k}+i} \cdot b_{n_{k}+j} \cdot c\right)=0$. Thus we can find an infinite $W \subseteq \omega$ such that $\mu\left(\sum_{i \in W} b_{i}\right) \leqslant \frac{1}{4}$. It follows that $\mu(\llbracket \tau \cap W=\varnothing \rrbracket) \geqslant \frac{1}{4}$.

Now let us see that $\infty$-Uncrowded $\left(\omega_{1}\right)$ does not imply Baire $\left(\omega_{1}\right)$.

Definition. Bounded ( $\kappa)$ iff $\forall F \in\left[\omega^{\omega}\right]^{<\kappa} \exists g \in \omega^{\omega} \forall f \in F \forall{ }^{\infty} n f(n)<g(n)$.

Definition. Weak Bounded ( $\kappa)$ iff $\forall F \in[\omega]^{<\kappa}, \exists g \in \omega^{\omega} \forall f \in F \exists^{\infty} n f(n)<$ $g(n)$.

LEMMA 1. $(1)^{2}$ Bounded $(\kappa) \Rightarrow$ Independent $(\kappa)$.

(2) Independent $(\kappa)+$ Weak Bounded $(\kappa) \Rightarrow \infty$-Uncrowded $(\kappa)$.

Proof. (1) This is a generalization of Theorem 2 of Solomon (1977). Given $B \in\left[[\omega]^{\omega}\right]^{<\kappa}$ define for each $X \in B, g_{X} \in \omega^{\omega}$ by letting $g_{X}(n)$ be least element of $B$ greater than $n$. By Bounded $(\kappa)$ find $f \in \omega^{\omega}$ which eventually dominates each $g_{X}$ for $X \in B$. Let

$$
Z=\bigcup_{n<\omega}[f(2 n), f(2 n+1)]
$$

Then for all $X \in B$,

$$
|X \cap Z|=|X-Z|=\omega .
$$

(2) Given $B \in\left[[\omega]^{\omega}\right]^{\leqslant \kappa}$ find using Independent ( $)^{\text {a }}$ a sequence $Z_{n+1} \subseteq Z_{n}$ such that $Z_{n}$ is $n$-uncrowded and for all $X \in B,\left|Z_{n} \cap X\right|=\omega$. For each $X \in B$ define $f_{x} \in \omega^{\omega}$ by requiring that for each $n<\omega,\left[n, f_{x}(n)\right) \cap X \cap Z_{n} \neq \varnothing$. By Weak

${ }^{2}$ This was discovered also by P. Nyikos, F. Galvin, and G. Gruenhage. 
Bounded ( $\kappa$ ) let $g \in \omega^{\omega}$ infinitely often dominate each $f_{x}$ and put

$$
Z=\cup\left\{[n, g(n)) \cap Z_{n} \mid n<\omega\right\} .
$$

In Laver's model (Laver (1976)), it is well known that Bounded $\left(\omega_{1}\right)$ holds but Baire $\left(\omega_{1}\right)$ fails.

Some remarks and questions. Can one drop the set $X$ from Different $(\kappa)$ or the family $B$ from Equal $(\kappa)$ ? If one changes the definition of Weak Bounded $(\kappa)$ to

$$
\forall F \in\left[\omega^{\omega}\right]^{\leqslant \kappa} \forall B \in\left[[\omega]^{\omega}\right]^{\leqslant \kappa} \exists g \in \omega^{\omega} \forall X \in B \forall f \in F\left(\exists^{\infty} n \in X f(n)<g(n)\right),
$$

one gets an equivalent property (Roitman (1979)).

The property Bounded $(\kappa)$ was defined and studied by Rothberger (1939, 1941, 1952). See also R. Solomon (1977). The property Weak Bounded ( $\kappa$ ) has been studied in connection with $p$-points, see Ketonen (1976) and in connection with box products, see Williams (1976), Roitman (1979), and van Douwen (1981).

For some models in which Independent $\left(\omega_{1}\right)$ fails, see Baumgartner and Laver (1979) and Kunen (1980) (in particular Exercises A10, A12, and A13 on p. 289).

Can the least $\kappa$ for which Independent $(\kappa)$ fails have cofinality $\omega$ ?

\section{REFERENCES}

J. Baumgartner and R. Laver, Iterated perfect set forcing, Ann. Math. Logic 17 (1979), 271-288.

G. Fichtenholz and L. Kantorovich, Sur les opérations linéaires dans l'espace des fonctions bornées, Studia Math. 5 (1934), 69-98.

E. van Douwen, Covering and separation properties of box products, in Surveys in General Topology, G. M. Reed (ed.), Academic Press, New York, 1981.

R. Laver, On the consistency of Borel's conjecture, Acta Math. 137 (1976), 151-169.

J. Ketonen, On the existence of p-points in the Stone-Čech compactification of the integers, Fund. Math. 92 (1976), $91-94$.

K. Kunen, Set theory, an introduction to independence proofs, North-Holland, Amsterdam, 1980.

K. Kuratowski, Topology, vol. 1, Academic Press, New York, 1966.

A. Miller, Some properties of measure and category, Trans. Amer. Math. Soc. 266 (1981), 93-114; Corrections, Trans. Amer. Math. Soc. 271 (1982), 347-348.

275-288. The Baire category theorem and cardinals of countable cofinality, J. Symbolic Logic 47 (1982),

R. Price, Ph.D. Dissertation, Univ. of Wisconsin, Madison, 1976.

L. Roitman, More paracompact box products, Proc. Amer. Math. Soc. 74 (1979), 171-175.

F. Rothberger, Sur un ensemble de première catégorie qui est dépourvu de la propriété $\lambda$, Fund. Math. 32 (1939), 294-300.

, Sur les familles indénombrables de suites de nombres naturels et les problèmes concernant la propriété C, Proc. Cambridge Philos. Soc. 37 (1941), 109-126.

, On the property $C$ and a problem of Hausdorff, Canad. J. Math. 4 (1952), 111-116.

W. Sierpinski, Sur une decomposition d'ensembles, Monatsh. Math. 35 (1928), 239-242.

R. Solomon, Families of sets and functions, Czechoslovak Math. J. 27 (1977), 556-559.

S. Williams, Is $\square^{\omega}(\omega+1)$ paracompact?, Topology Proc. 1 (1976), 141-146. 